OPEN ACCESS

Edited by:

Tom Carr,

Michigan State University,

United States

Reviewed by:

Ryoko Sasamoto,

Dublin City University, Ireland

Eleonora Borelli,

University of Modena and Reggio

Emilia, Italy

${ }^{*}$ Correspondence:

Keisuke Irie

irie.keisuke.8n@kyoto-u.ac.jp

Specialty section:

This article was submitted to

Cognition

a section of the journal

Frontiers in Psychology

Received: 11 June 2021 Accepted: 02 September 2021 Published: 22 September 2021

Citation:

Irie $K$, Zhao S, Okamoto $K$ and Liang N (2021) Examining the Effect of Adverbs and Onomatopoeia on

Physical Movement.

Front. Psychol. 12:723602. doi: 10.3389/fpsyg.2021.723602

\section{Examining the Effect of Adverbs and Onomatopoeia on Physical Movement}

\author{
Keisuke Irie ${ }^{1 *}$, Shuo Zhao ${ }^{2}$, Kazuhiro Okamoto ${ }^{3}$ and Nan Liang ${ }^{1}$ \\ ${ }^{1}$ Cognitive Motor Neuroscience, Department of Human Health Sciences, Graduate School of Medicine, Kyoto University, \\ Kyoto, Japan, ${ }^{2}$ School of Psychology, Shenzhen University, Shenzhen, China, ${ }^{3}$ Department of Rehabilitation, Faculty \\ of Health Science, Fukui Health Science University, Fukui, Japan
}

Introduction: The effect of promoting a physical reaction by the described action is called the action-sentence compatibility effect (ACE). It has been verified that physical motion changes depending on the time phase and grammatical expression. However, it is unclear how adverbs and onomatopoeia change motion simulations and subsequent movements.

Methods: The subjects were 35 healthy adults (11 females; mean age 21.3). We prepared 20 sentences each, expressing actions related to hands and feet. These were converted into 80 sentences (stimulus set A), with the words "Slow" or "Quick" added to the words related to the speed of movement, and 80 sentences (stimulus set B) with the words "Fast" and onomatopoeia "Satto" added. Additionally, 20 unnatural sentences were prepared for each stimulus set as pseudo sentences. Choice reaction time was adopted; subjects pressed the button with their right hand only when the presented text was correctly understood (Go no-go task). The reaction time (RTs) and the number of errors (NoE) were recorded and compared.

Results: As a result of a two-way repeated ANOVA, an interaction effect (body parts $\times$ words) was observed in RTs and NoE in set A. "Hand and Fast" had significantly faster RTs than "Hand and Slow" and "Foot and Fast." Furthermore, "Hand and Fast" had a significantly higher NoE than others. In set $\mathrm{B}$, the main effects were observed in both RTs and NoE. "Hand" and "Satto" had significantly faster RTs than "Foot" and "Quick," respectively. Additionally, an interaction effect was observed in NoE, wherein "Foot and Satto" was significantly higher than "Hand and Satto" and "Foot and Quick."

Conclusion: In this study, the word "Fast" promoted hand response, reaffirming ACE. The onomatopoeia "Satto" was a word that conveys the speed of movement, but it was suggested that the degree of understanding may be influenced by the body part and the attributes of the subject.

Keywords: ACE, action verbs, onomatopoeia, grounded cognition, adverbs (intensifying) 


\section{INTRODUCTION}

In sports and rehabilitation, motor learning is promoted by explaining how the movement needs to be performed to the individual and providing knowledge of results (KR). Although KR is useful for motor correction, studies have shown that excessive dependence on KR negatively impacts subsequent performance (Badets and Blandin, 2010; Chiviacowsky et al., 2010) by making it less likely for internal feedback to develop. Therefore, it is necessary to promote internal feedback by gradually reducing the frequency of KR. Recent suggestions state that a combination of coaching and exercise effectively improves both athletic and psychosocial performance (Kivelä et al., 2014; Ovans et al., 2018). Thus, language can be used to affect the movements of individuals in many situations.

"Onomatopoeia" is a word that imitates the sounds made by people, animals, or nature. According to the Oxford English Dictionary, "onomatopoeia" is defined as "the formation of words from sounds associated with the thing named." These are well-established words with a long history, dating back at least four centuries (Assaneo et al., 2011). The term onomatopoeia is also used in motion instruction to describe the motion's force, speed, and timing. In Japanese, examples include "Gutto" (Putting in a moment of effort), "Satto" ("In a flash"), and "Sotto (Doing something quietly)." It allows for the concise expression of meanings and images that are otherwise difficult to express; consequently, it has been documented as an important language learning and communication tool for young children (Laing, 2017). Onomatopoeia falls under the expressive aspect of communication, as it often conveys extremely vague impressions that are difficult to express purely as propositions (Blakemore, 2008, 2011, 2014). Sasamoto and Jackson (2016) have shown that communicators can use any available tools and that onomatopoeia results from attempts to replicate sensory experiences, especially through the use of similarity. However, few papers have examined whether onomatopoeia accurately conveys the intended information using experimental methods.

In the last two decades, many studies have investigated the association between sentence comprehension and sensorimotor representation. Grounded cognition proposes that modal simulations, body states, and situated actions underlie cognition, and the representation of concepts activates the same sensorymotor modalities that recognize and act on those concepts (Barsalou, 1999, 2008, 2010; Glenberg and Kaschak, 2002; Gallese and Lakoff, 2005). This simulation reflects the meaning of the words and the meaning and grammar of the whole sentence. For example, negative sentences undergo a two-step process in which the affirmative sentence is first simulated and then negated (Kaup et al., 2006, 2007). Furthermore, some studies have shown that the progressive form facilitates the response, but the past tense of the verb does not (Madden and Therriault, 2009; Bergen and Wheeler, 2010). Behavioral experiments have repeatedly reported that when an active sentence is judged to be meaningful, the physical movement or action associated with that sentence is promoted (Dudschig et al., 2012; Diefenbach et al., 2013; Ibáñez et al., 2013). Specifically, subjects pressed one of two buttons placed near or far from a given body after determining whether a sentence made sense. In some cases, the sentence included moving the hand closer to or further away from the body. For example, the sentence "he took off his glasses" represented the action of "moving his hands away from his body." Conversely, the sentence "he put on his glasses" represented the action of "moving his hands closer to his body." When the action described in a sentence is consistent with the action the subject must perform to respond to that sentence, the response is faster than when the actions are not consistent (Glenberg and Kaschak, 2002; Zwaan et al., 2012). This behavioral effect, known as the action-sentence compatibility effect (ACE), has been observed in multiple languages, including English (Borreggine and Kaschak, 2006), French (Boulenger et al., 2006), Italian (Glenberg et al., 2008), and Japanese (Awazu, 2011). Thus, using ACE, it is possible to investigate the influence of grammatical aspects of a sentence, in addition to the nouns and verbs used. We use the adverbs "Slow" or "Quick" when referring to the speed of movement in our daily lives. However, the effects of adverbs used in motion commands and onomatopoeia with similar meanings on physical responses have not been tested.

Therefore, the objectives of this study are as follows: first, to examine whether the words "Slow" and "Fast," which describe the speed of the action indicated by the verb, affect the simulation of movement. In other words, we expected that words related to hand movement would facilitate both "Slow" and "Fast" hand responses, while words related to foot movement would not produce ACE; thus, it would not change the results. Second, we tested the usefulness of the onomatopoeic word "Satto" to promote speed of movement. We hypothesized that the reaction time would be shorter for "Satto" than for "Quick" in words related to hand movements. To clarify these hypotheses, we conducted a single experiment consisting of two consecutive sub-experiments (stimulus set $\mathrm{A}$ and stimulus set $\mathrm{B}$ ).

\section{MATERIALS AND METHODS}

\section{Participants}

This cross-sectional study included 35 healthy students as participants (11 females, mean age: $21.3 \pm 2.4$ years) from Kyoto University. All participants were informed about the procedures involved in the experiments, and those who provided their informed consent were included. The exclusion criteria were as follows: (1) those who were not native Japanese speakers and (2) those who could not perform reading comprehension or press a button, owing to cognitive or motor difficulties [IQ70 or higher was confirmed by the Japanese Adult Reading Test (Matsuoka et al., 2006)]. In addition, right-handedness was assessed using the Edinburgh Handedness Questionnaire (Oldfield, 1971). The study was approved by the Ethics Committee of Kyoto University Graduate School and the Faculty of Medicine (approval number R2188-1).

\section{Apparatus}

The experiments were conducted using a computer (Panasonic, CF-SV) and a 17-inch display monitor (MITSUBISHI and RDT20IWM). Software (E-Prime 3.0: Psychology Software 
Tools, Inc.) was used to control stimulus presentation and record participants' responses. First, participants placed their right index finger on a mark $20 \mathrm{~cm}$ away from the keyboard. Next, a sentence was presented at random. The participant was then instructed to press the button only if they understood the sentence.

\section{Stimulus}

We prepared 20 types of sentences expressing actions related to each hand and foot in Japanese and converted them into two different ones: one that used the word "Fast" (e.g., "picking it up fast," "stand up fast") and another using the word "Slow" (e.g., "picking it up slowly," "sit down slowly"), that is, 40 sentences using the word "Fast" and 40 using the word "Slow." In addition to these 80 sentences, we prepared 20 pseudo-sentences with a meaningless combination of nouns and verbs (e.g., "Pour hand"). These 100 sentences were designated as stimulus set A. In stimulus set $B$, which was created to examine the usefulness of onomatopoeia, 20 prepared sentences were converted into "Satto" (which means "in a flash" in Japanese) and "Quick," and 80 types of sentences were created in the same manner as above. Additionally, as in set A, we prepared 20 pseudo-sentences that used the Japanese terms for "Fast" and "Quick" ("Subayaku" and "Hayaku," respectively). "Quick" denotes actions performed with rapid velocity within a short duration, whereas "Fast" denotes actions performed with rapid velocity within a long duration. Different combinations of words were applied to a set of sentences so that the number of letters in comparison and the control groups would be the same.

\section{Procedure}

Participants were tested after being informed about the procedures involved in the experiment and a simulated practice of the experiment had been conducted (Figure 1). The test session was composed of two 100-trial blocks, with the order of the trial blocks randomized. For each trial, a fixation cross point was first presented in the center of the screen for 3,000 ms. Subsequently, sentences related to either hand or foot movements or pseudo-sentences were presented for up to $4,000 \mathrm{~ms}$. The order of the stimuli was randomized within each block. The participants took a break between blocks for approximately $3 \mathrm{~min}$. In each trial, the participants were required to judge whether a stimulus sentence was meaningful or not. It was treated as a go/no-go task-they were asked to press the button only if they could understand the sentence (i.e., they were able to perform the action mentioned in the sentence). Finally, at the end of the experiment, the participants were asked which of the two terms- "Satto" or "Quick"-was easier for them to understand.

\section{Analysis}

All statistical analyses were conducted using IBM SPSS Statistics version 26 (IBM, Armonk, NY, United States). Trials with reaction times of $<300 \mathrm{~ms}$ and trials with reaction times greater than three standard deviations were excluded. This is because it is highly likely that the reaction time is fast considering that the sentence is not read; conversely, the reaction time could be slow for some reason other than reading. The main outcomes were reaction times (RTs) and number of errors (NoE), and a two-way repeated analysis of variance (ANOVA) was performed on words (Slow-Fast and Quick-Satto) and body parts (hand-related condition and foot-related condition). If a two-way interaction was significant, a follow-up simple main effect (i.e., assessing the effect of each independent variable at each level of the other independent variable) analysis was conducted $(p<0.05)$.

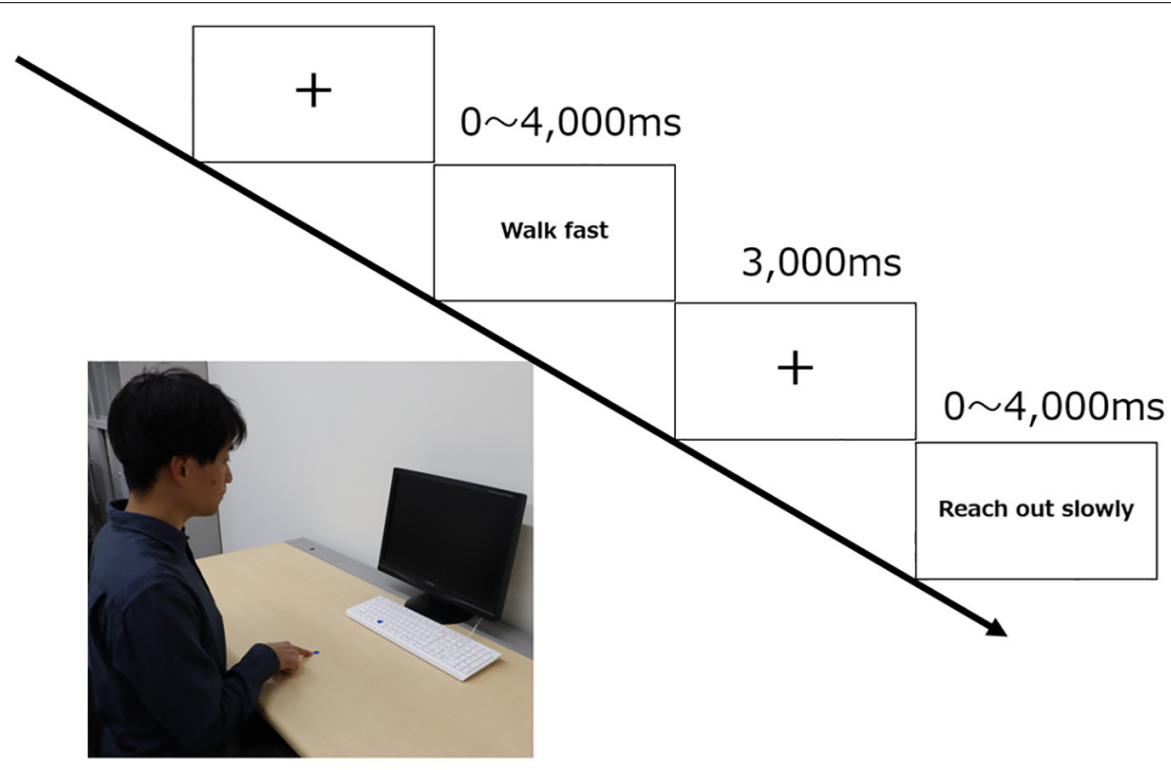

FIGURE 1 | Experiment protocol followed during the study. Participants placed the index finger of their right hand on a mark $20 \mathrm{~cm}$ away from the keyboard. A sentence was presented at random, and the participant was instructed to press the button only if they understood the sentence. 


\section{RESULTS}

The mean RTs and NoE for stimulus sets A and B are listed in Table 1.

\section{Effect of Action-Sentence Compatibility on Reaction Times}

The two-way repeated ANOVA conducted for the RTs for stimulus set A indicated a main effect for words, so that "Fast" produced faster RTs than "Slow" $[F(1,34)=79.58, p<0.001$ $\left.\eta p^{2}=0.70\right]$. Furthermore, a main effect was observed for body parts, so that "Hand" produced faster RTs than "Foot" $\left[F(1,34)=12.47, p=0.001, \eta p^{2}=0.27\right]$. Moreover, the interaction effect (body parts and words) was significant $[F(1,34)=24.07$, $p<0.001$, $\eta$ p $2=0.41]$. In the post hoc test, "Hand and Fast" showed significantly faster RTs than "Hand and Slow" and "Foot and Fast" $(p<0.05$, Figure 2).

In stimulus set B, a main effect was observed for words-such that "Satto" produced faster RTs than "Quick" $[F(1,34)=36.34$, $p<0.001$, $\eta p^{2}=0.52$ ) -and body parts—such that "Hand" produced faster RTs than "Foot" $[F(1,34)=5.29, p=0.028$, $\left.\eta p^{2}=0.36\right]$. However, no significant interaction effect was observed (Figure 3).

\section{Effect of Action-Sentence Compatibility on Number of Errors}

The two-way repeated ANOVA conducted for the NoE in stimulus set A indicated a main effect for words $[F(1,34)=17.37$, $\left.p<0.001, \eta p^{2}=0.34\right]$. Furthermore, the interaction effect was significant $\left[F(1,34)=12.11, p=0.001, \eta p^{2}=0.26\right]$. In the post hoc test, "Hand and Fast" was found to have a significantly higher NoE than the other conditions ( $p<0.05$, Figure 2 ).

In stimulus set $\mathrm{B}$, no significant main effects were observed $[F(1,34)=0.03, p=0.873]$. However, the interaction effect was found to be significant $\left[F(1,34)=18.51, p<0.001, \eta p^{2}=0.3\right]$. In the post hoc test, NoE was significantly higher in "Foot and Satto" than "Hand and Satto" and "Foot and Quick" ( $p<0.05$, Figure 3).

\section{Understanding Onomatopoeia}

Based on the survey conducted at the end of the experiment, it was observed that 19 participants (54.3\%) felt onomatopoeia

TABLE 1 | Mean reaction times (RTS) and number of errors (NoE) observed in each experimental condition.

\begin{tabular}{lllll}
\hline Stimulus set & $\begin{array}{l}\text { Body parts } \\
\text { (Effector) }\end{array}$ & $\begin{array}{l}\text { Words } \\
\text { (Effector) }\end{array}$ & RTs (in ms) (SE) & NoE (SE) \\
\hline Set A & Hand & Slow & $1710.9(70.9)$ & $0.5(0.1)$ \\
& & Fast & $1414.5(39.5)$ & $1.4(0.1)$ \\
& Foot & Slow & $1749.3(65.9)$ & $0.7(0.2)$ \\
& \multirow{2}{*}{ Set B } & Fast & $1845.7(81.4)$ & $0.8(0.3)$ \\
& Hand & Satto & $1517.2(43.2)$ & $1.4(0.2)$ \\
& \multirow{2}{*}{ Foot } & Quick & $1595.4(50.6)$ & $1.1(0.2)$ \\
& & Satto & $1609.8(54.3)$ & $3.0(0.5)$ \\
& & Quick & $1665.8(55.1)$ & $1.7(0.3)$
\end{tabular}

("Satto") was easier to understand, while 16 participants (45.7\%) felt that the word "Quick" was easier to understand.

\section{DISCUSSION}

We hypothesized that ACE, which arises from an understanding of the words "Fast" and "Slow," would be observed only in the case of sentences related to hand movements. Additionally, we hypothesized that the onomatopoeia "Satto" would generate a faster reaction time than "Quick" when comprehending handrelated sentences. Both hypotheses were partially confirmed; in the case of hand-related sentences, the word "Fast" led to significantly faster RTs than "Slow." Further, although "Satto" led to significantly faster RTs than "Quick" in the case of both handand foot-related movements, no significant interaction effect was observed. In addition to these findings, we obtained interesting results regarding NoE; the combinations of the words "Hand and Fast" and "Foot and Satto" led to significantly higher NoE than the other combinations.

In stimulus set $\mathrm{A}$, we used adverbs with opposite meanings, "Slow" and "Fast," to test whether different adverbs showed ACE. It has been reported that familiarity with words is a factor that affects comprehension (Pulvermüller et al., 1999). According to the NTT database series, "Lexical Features of Japanese," "Slow" has a higher degree of intimacy and mental image than "Fast" and is considered to be easier to understand. However, in this study, the reaction time of "Fast" was faster only for words related to "Hands." This may be because the participants understood the word by simulating the action, which facilitated the same physical reaction. In other words, the ACE was confirmed for adverbs as well. Motor imagery studies have previously reported that cortical neurons are linked to task-specific activation that can inhibit or promote slow(er) motor pathways (Keller et al., 2018). It has also been suggested that both slow and fast movements involve an automated system (Cressman et al., 2010). However, in this study, the word "Fast" promoted hand response, but "Slow" did not inhibit/slow down the response. A possible explanation for this may be the influence of the experimental environment. Specifically, we can unconsciously change our movements by simply observing the surrounding objects and environment (McDannald et al., 2018). This concept is called "affordance" (Osiurak et al., 2017) and has been demonstrated using a variety of imaging techniques, including fMRI (Grafton et al., 1997; Grèzes et al., 2003). Since the reaction task generally necessitates a rapid reaction, such an experimental condition may promote the entire reaction.

Regarding the accuracy of the response, the "Hand and Fast" combination was observed to be significantly less accurate than the other combinations. Classical studies by Fitts (1954), Woodworth (1899), and others have shown a trade-off between speed and accuracy during exercise (Plamondon and Alimi, 1997). Even in a language processing task, it has been shown that errors increase when the go signal is issued immediately before the reaction (Mirabella et al., 2012). In other words, shorter word processing time increases the likelihood of an error occurrence. This study results can form the basis for suggesting that there is 

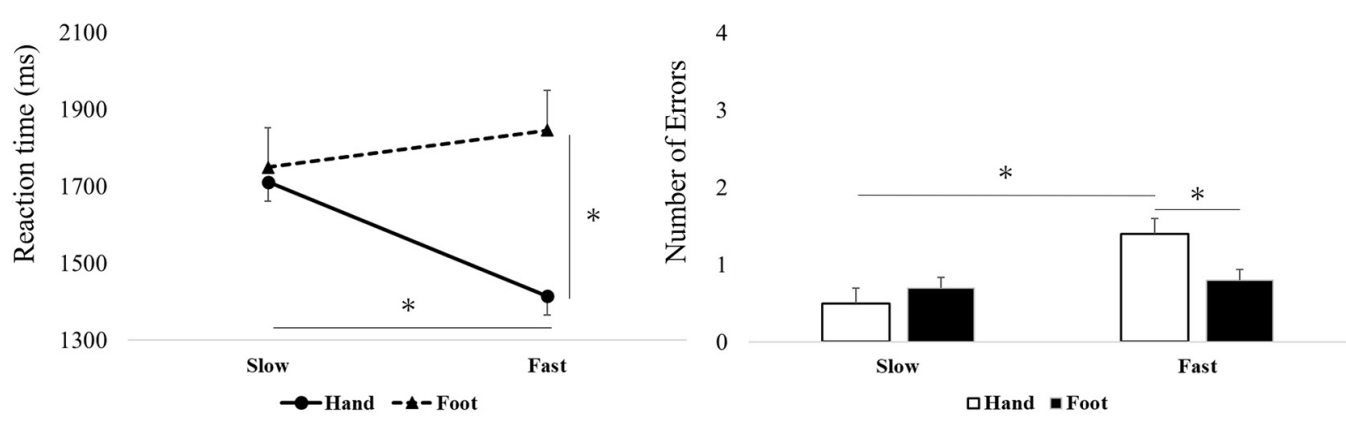

FIGURE 2 | Reaction times (RTs) and number of errors (NoE) in "Slow or Fast" and "Hand or Foot" combinations. Based on the post hoc test, "Hand and Fast" showed significantly faster RTs, as well as a significantly greater NoE, than combinations involving "Foot" and "Slow" * $p<0.05$.

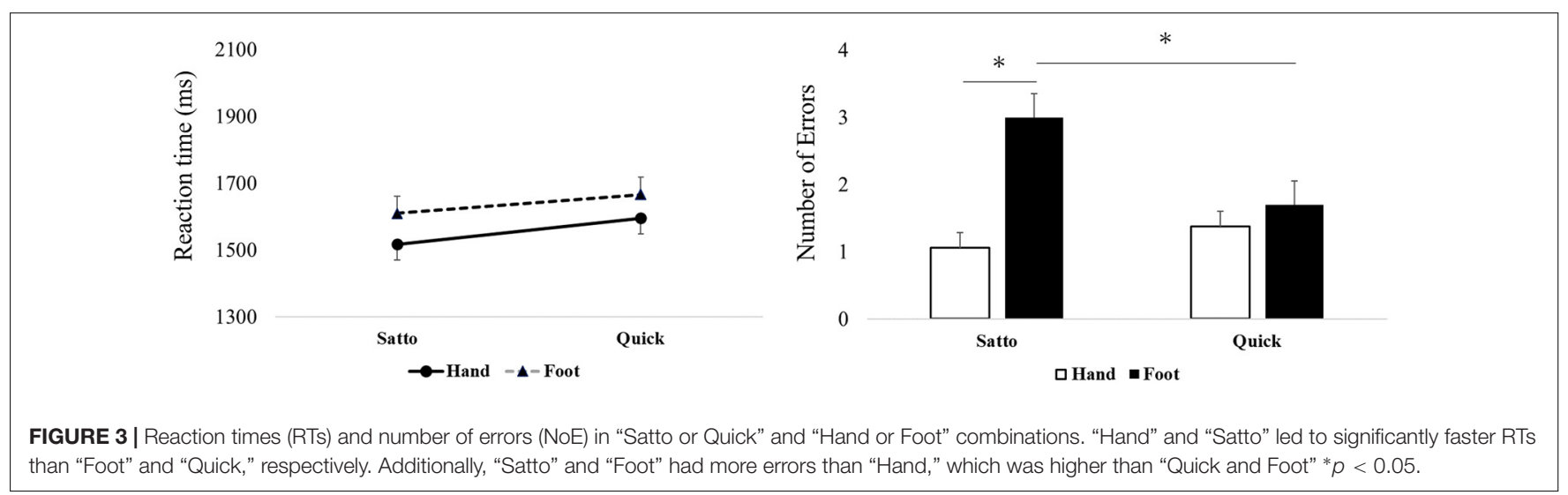

a trade-off between speed and accuracy, not only in behavior, but also at the cognitive level.

In stimulus set B, we used the onomatopoeia "Satto," synonymous with "Quick," to test whether the onomatopoeia facilitates a physical response. The onomatopoeia "Satto," frequently used in motion guidance, had a significantly faster reaction time than "Quick," even though no interaction effect was observed. Thus, "Satto" is more likely to promote action than "Quick." Many studies have consistently demonstrated the benefits of symbolism in language learning, that is, using seemingly non-arbitrary linguistic or gestural cues that are symbolically linked to the word's meaning (Imai et al., 2008; Asano et al., 2015; Lockwood et al., 2016). Onomatopoeia is believed to be present in most languages globally, and psychological experiments have shown that sound symbols in one language can be understood by speakers of another language (Svantesson, 2017). An fMRI study reported the involvement of the right posterior superior temporal sulcus, which is thought to be useful for understanding non-verbal communication, such as sound, in understanding onomatopoeia (Kanero et al., 2014). Thus, onomatopoeia is a word that can be understood through its sensory (e.g., auditory) features, although its exact meaning may be difficult to understand. This was confirmed by our study findings, wherein $54 \%$ of the participants indicated that "Satto" was easier to understand, as opposed to "Quick." This provides a possible explanation for the absence of interaction effect observed in the RTs between "Quick" and "Satto." Of particular interest is the finding that the NoE for "Foot and Satto" was lower than that observed for "Hand and Satto." No previous studies have shown that body parts affect the understanding of language and onomatopoeia. This result indicates that the understanding of onomatopoeia may be influenced by attributes, such as body parts and the subject's experience.

The current study has a few limitations. First, it was unclear whether ACE can also be observed when participants are instructed to react to the stimuli using the foot. Second, usual movement instruction is often carried out through auditory means; however, this study adopted visual stimulus presentation. Hence, the relationship between auditory understanding and behavior has not been clarified. Third, our study focused only on the onomatopoeia "Satto," which limits the generalizability of the finding to other onomatopoeia terms. Future research will need to develop protocols that involve the use of auditory stimuli and foot responses. In addition, it is necessary to improve the matching of different terms to body parts through prior investigation of the onomatopoeia used for movement.

\section{CONCLUSION}

We examined the effect of adverbs used in motion instruction on motion simulation and comprehension. "Fast" was found to promote motor responses, but can reduce language comprehension, leading to increased errors. The onomatopoeia 
"Satto" encourages more motor response than "Quick;" however, the degree of understanding of words may be affected by body parts. Therefore, it is necessary to confirm the participant's comprehension level of a word in movement guidance.

\section{DATA AVAILABILITY STATEMENT}

The original contributions presented in the study are included in the article/Supplementary Material, further inquiries can be directed to the corresponding author.

\section{ETHICS STATEMENT}

The studies involving human participants were reviewed and approved by the Kyoto University Graduate School and Faculty of Medicine (approval number R2188-1). The patients/participants provided their written informed consent to participate in this study.

\section{AUTHOR CONTRIBUTIONS}

KI and SZ designed the study. KI performed the experiment, analyzed the data, and wrote the manuscript. KI, KO, and NL

\section{REFERENCES}

Asano, M., Imai, M., Kita, S., Kitajo, K., Okada, H., and Thierry, G. (2015). Sound symbolism scaffolds language development in preverbal infants. Cortex 63, 196-205. doi: 10.1016/j.cortex.2014.08.025

Assaneo, M. F., Nichols, J. I., and Trevisan, M. A. (2011). The anatomy of onomatopoeia. PLoS One 6:e28317. doi: 10.1371/journal.pone.0028317

Awazu, S. (2011). The action-sentence compatibility effect in Japanese sentences. Percept. Mot. Skills 113, 597-604. doi: 10.2466/28.PMS.113.5.597-604

Badets, A., and Blandin, Y. (2010). Feedback schedules for motor-skill learning: the similarities and differences between physical and observational practice. J. Mot. Behav. 42, 257-268. doi: 10.1080/00222895.2010.497512

Barsalou, L. W. (1999). Perceptions of perceptual symbols. Behav. Brain Sci. 22, 637-660. doi: 10.1017/S0140525X99532147

Barsalou, L. W. (2008). Grounded cognition. Ann. Rev. Psychol. 59, 617-645. doi: 10.1146/annurev.psych.59.103006.093639

Barsalou, L. W. (2010). Grounded cognition: past, present, and future. Top. Cogn. Sci. 2, 716-724. doi: 10.1111/j.1756-8765.2010.01115.x

Bergen, B., and Wheeler, K. (2010). Grammatical aspect and mental simulation. Brain Lang. 112, 150-158. doi: 10.1016/j.bandl.2009.07.002

Blakemore, D. (2008). Apposition and affective communication. Lang. Lit. 17, 37-57. doi: 10.1177/0963947007085054

Blakemore, D. (2011). On the descriptive ineffability of expressive meaning. J. Pragmat. 18, 101-118. doi: 10.1016/j.pragma.2011.08.003

Blakemore, D. (2014). Slurs and expletives: a case against a general account of expressive meaning. Lang. Sci. 52, 22-35. doi: 10.1016/j.langsci.2014. 06.018

Borreggine, K. L., and Kaschak, M. P. (2006). The action-sentence compatibility effect: it's all in the timing. Cogn. Sci. 30, 1097-1112. doi: 10.1207/ s15516709cog0000_91

Boulenger, V., Roy, A. C., Paulignan, Y., Deprez, V., Jeannerod, M., and Nazir, T. A. (2006). Cross-talk between language processes and overt motor behavior in the first $200 \mathrm{msec}$ of processing. J. Cogn. Neurosci. 18, 1607-1615. doi: 10.1162/jocn.2006.18.10.1607 interpreted the results. SZ checked the text and created part of the figure. NL checked and revised the manuscript. All authors approved the final version of the manuscript.

\section{FUNDING}

This work was partially supported by the JSPS KAKENHI Grantin-Aid for Early-Career Scientists (Grant No. 19K19832) from the Japan Society for the Promotion of Science, which was used to purchase the equipment needed for the experiment, and SPIRITS 2021 of Kyoto University, which was used for English proofreading and submission costs.

\section{ACKNOWLEDGMENTS}

We would like to thank Editage (www.editage.com) for English language editing.

\section{SUPPLEMENTARY MATERIAL}

The Supplementary Material for this article can be found online at: https://www.frontiersin.org/articles/10.3389/fpsyg. 2021.723602/full\#supplementary-material

Chiviacowsky, S., Campos, T., and Domingues, M. R. (2010). Reduced frequency of knowledge of results enhances learning in persons with Parkinson's disease. Front. Psychol. 1:226. doi: 10.3389/fpsyg.2010.00226

Cressman, E. K., Cameron, B. D., Lam, M. Y., Franks, I. M., and Chua, R. (2010). Movement duration does not affect automatic online control. Hum. Mov. Sci. 29, 871-881. doi: 10.1016/j.humov.2010.07.001

Diefenbach, C., Rieger, M., Massen, C., and Prinz, W. (2013). Action-sentence compatibility: the role of action effects and timing. Front. Psychol. 4:272. doi: 10.3389/fpsyg.2013.00272

Dudschig, C., Lachmair, M., De La Vega, I., De Filippis, M., and Kaup, B. (2012). Do task-irrelevant direction-associated motion verbs affect action planning? Evidence from a Stroop paradigm. Mem. Cogn. 40, 1081-1094. doi: 10.3758/ s13421-012-0201-9

Fitts, P. M. (1954). The information capacity of the human motor system in controlling the amplitude of movement. J. Exp. Psychol. 47, 381-391. doi: 10. 1037/h0055392

Gallese, V., and Lakoff, G. (2005). The brain's concepts: the role of the sensorymotor system in conceptual knowledge. Cogn. Neuropsychol. 22, 455-479. doi: $10.1080 / 02643290442000310$

Glenberg, A. M., and Kaschak, M. P. (2002). Grounding language in action. Psychon. Bull. Rev. 9, 558-565. doi: 10.3758/bf03196313

Glenberg, A. M., Sato, M., Cattaneo, L., Riggio, L., Palumbo, D., and Buccino, G. (2008). Processing abstract language modulates motor system activity. Q. J. Exp. Psychol. 61, 905-919. doi: 10.1080/17470210701625550

Grafton, S. T., Fadiga, L., Arbib, M. A., and Rizzolatti, G. (1997). Premotor cortex activation during observation and naming of familiar tools. Neuroimage 6, 231-236. doi: 10.1006/nimg.1997.0293

Grèzes, J., Tucker, M., Armony, J., Ellis, R., and Passingham, R. E. (2003). Objects automatically potentiate action: an fMRI study of implicit processing. Eur. J. Neurosci. 17, 2735-2740. doi: 10.1046/j.1460-9568.2003.02695.x

Ibáñez, A., Cardona, J. F., Dos Santos, Y. V., Blenkmann, A., Aravena, P., Roca, M., et al. (2013). Motor-language coupling: direct evidence from early Parkinson's disease and intracranial cortical recordings. Cortex 49, 968-984. doi: 10.1016/j. cortex.2012.02.014 
Imai, M., Kita, S., Nagumo, M., and Okada, H. (2008). Sound symbolism facilitates early verb learning. Cognition 109, 54-65. doi: 10.1016/j.cognition.2008.07.015

Kanero, J., Imai, M., Okuda, J., Okada, H., and Matsuda, T. (2014). How sound symbolism is processed in the brain: a study on Japanese mimetic words. PLoS One 9:e97905. doi: 10.1371/journal.pone.0097905

Kaup, B., Lüdtke, J., and Zwaan, R. A. (2006). Processing negated sentences with contradictory predicates: is a door that is not open mentally closed? J. Pragmat. 38, 1033-1050. doi: 10.1016/j.pragma.2005.09.012

Kaup, B., Lüdtke, J., and Zwaan, R. A. (2007). Experiential simulations of negated text information. Q. J. Exp. Psychol. 60, 976-990. doi: 10.1080/ 17470210600823512

Keller, M., Taube, W., and Lauber, B. (2018). Task-dependent activation of distinct fast and slow(er) motor pathways during motor imagery. Brain Stimul. 11, 782-788. doi: 10.1016/j.brs.2018.02.010

Kivelä, K., Elo, S., Kyngäs, H., and Kääriäinen, M. (2014). The effects of health coaching on adult patients with chronic diseases: a systematic review. Patient Educ.Couns. 97, 147-157. doi: 10.1016/j.pec.2014.07.026

Laing, C. E. (2017). A perceptual advantage for onomatopoeia in early word learning: evidence from eye-tracking. J. Exp. Child Psychol. 161, 32-45. doi: 10.1016/j.jecp.2017.03.017

Lockwood, G., Dingemanse, M., and Hagoort, P. (2016). Sound-symbolism boosts novel word learning. J. Exp. Psychol. 42, 1274-1281. doi: 10.1037/xlm0000235

Madden, C. J., and Therriault, D. J. (2009). Verb aspect and perceptual simulations. Q. J. Exp. Psychol. 62, 1294-1302. doi: 10.1080/17470210802696088

Matsuoka, K., Uno, M., Kasai, K., Koyama, K., and Kim, Y. (2006). Estimation of premorbid IQ in individuals with Alzheimer's disease using Japanese ideographic script (Kanji) compound words: japanese version of National Adult Reading Test. Psychiatry Clin. Neurosci. 60, 332-339. doi: 10.1111/j.1440-1819. 2006.01510.x

McDannald, D. W., Mansour, M., Rydalch, G., and Bolton, D. A. E. (2018). Motor affordance for grasping a safety handle. Neurosci. Lett. 683, 131-137. doi: 10.1016/j.neulet.2018.05.040

Mirabella, G., Iaconelli, S., Spadacenta, S., Federico, P., and Gallese, V. (2012). Processing of hand-related verbs specifically affects the planning and execution of arm reaching movements. PLoS One 7:e35403. doi: 10.1371/journal.pone. 0035403

Oldfield, R. C. (1971). The assessment and analysis of handedness: the Edinburgh inventory. Neuropsychologia 9, 97-113. doi: 10.1016/0028-3932(71)90067-4
Osiurak, F., Rossetti, Y., and Badets, A. (2017). What is affordance? 40 years later. Neurosci. Biobehav. Rev. 77, 403-417. doi: 10.1016/j.neubiorev.2017.04.014

Ovans, J. A., Hooke, M. C., Bendel, A. E., and Tanner, L. R. (2018). Physical therapist coaching to improve physical activity in children with brain tumors: a pilot study. Pediatr. Phys. Ther. 30, 310-317. doi: 10.1097/PEP. 0000000000000531

Plamondon, R., and Alimi, A. M. (1997). Speed/accuracy trade-offs in targetdirected movements. Behav. Brain Sci. 20, 279-303; discussion 303-249.

Pulvermüller, F., Lutzenberger, W., and Preissl, H. (1999). Nouns and verbs in the intact brain: evidence from event-related potentials and high-frequency cortical responses. Cereb. Cortex 9, 497-506. doi: 10.1093/cercor/9.5.497

Sasamoto, R., and Jackson, R. (2016). Onomatopoeia - showing-word or sayingword? relevance theory, lexis, and the communication of impressions. Lingua 175, 36-53. doi: 10.1016/j.lingua.2015.11.003

Svantesson, J. O. (2017). Sound symbolism: the role of word sound in meaning. Wiley Interdiscip. Rev. Cogn. Sci. 8:e1441. doi: 10.1002/wcs.1441

Woodworth, R. S. (1899). Accuracy of voluntary movement. Psychol. Rev. 3, 1-114.

Zwaan, R. A., Van Der Stoep, N., Guadalupe, T., and Bouwmeester, S. (2012). Language comprehension in the balance: the robustness of the actioncompatibility effect (ACE). PLoS One 7:e31204. doi: 10.1371/journal.pone. 0031204

Conflict of Interest: The authors declare that the research was conducted in the absence of any commercial or financial relationships that could be construed as a potential conflict of interest.

Publisher's Note: All claims expressed in this article are solely those of the authors and do not necessarily represent those of their affiliated organizations, or those of the publisher, the editors and the reviewers. Any product that may be evaluated in this article, or claim that may be made by its manufacturer, is not guaranteed or endorsed by the publisher.

Copyright $\odot 2021$ Irie, Zhao, Okamoto and Liang. This is an open-access article distributed under the terms of the Creative Commons Attribution License (CC BY). The use, distribution or reproduction in other forums is permitted, provided the original author(s) and the copyright owner(s) are credited and that the original publication in this journal is cited, in accordance with accepted academic practice. No use, distribution or reproduction is permitted which does not comply with these terms. 